Characterization of the chorismate mutase effector $(\mathrm{SsCm} 1)$ from Sclerotinia sclerotiorum

Dickman, M.B. Texas A\&M AgriLife Research

Yarden, $\mathrm{O}$. The Hebrew University of Jerusalem

Project award year: 2015

Three year research project 
The following is an abstract of our work, funded by BARD, which focused on the Sscm1 gene and corresponding protein in Sclerotinia sclerotiorum.

Prof. Marty Dickman, the US collaborator on this grant, passed away on December 2, 2018, following rapid deterioration in his health over the past 2 years. As the late phases of his disease imposed significant difficulties, some of our joint work has, sadly, not been completed. Marty was a brilliant and passionate scientist, a longtime colleague and a close friend.

Sclerotinia sclerotiorum is a hemibiotrophic, phytopathogenic, filamentous ascomycete fungus. It is a cosmopolitan plant pathogen with an extremely wide range of hosts ( $>400$ species), primarily dicots. S. sclerotiorum causes considerable damage in economically important crops [annual multimillion dollar losses, has proven difficult to control (culturally or chemically) and host resistance to this fungus has generally been inadequate. While historically, S. sclerotiorum has been classified as a prototypical necrotrophic fungus, although our recent BARD-funded work questions this assertion. Virulence of this aggressive pathogen is bolstered by a wide array of cell wall degrading enzymes and secondary metabolites. A primary virulence determinant associated with Sclerotinia virulence is the production of millimolar concentrations of the non-specific phytotoxin, oxalic acid. It is well established that phytopathogens secrete proteins and small molecules that interact with host cells and play a critical role in disease development. Such molecules, collectively known as effectors, are defined in broad terms as molecules (usually small) that are secreted by the pathogen, are plant induced and modulate host responses. Plant resistance against some pathogens can be mediated by recognition of such effectors. Alternatively, effectors can interfere with plant defense. As part of an ongoing hostpathogen arms race, some effectors are recognized by the host plant $\mathrm{R}$ gene-encoded receptors that can culminate in a programmed cell death (PCD) resistant response known as Effector Triggered Immunity. With the exception of host-selective toxin producing necrotrophs, the field of fungal effectors has generally concentrated on biotrophs and hemibiotrophs and the situation with "classical necrotrophs” such as S. sclerotiorum are for the most part not well understood. Several years ago an effector with a novel mode of action was isolated from the corn smut pathogen and model biotroph, Ustilago maydis. 
The effector gene (cmu1) encodes a secreted chorismate mutase, the expression of which was shown to be required for full virulence. Chorismate mutases are part of the shikimate pathway, a central metabolic route in plants and microbes. The shikimic pathway is essential for the synthesis of a number of metabolites including phytoalexins, aromatic amino acids and glucosinolates, among others. These aromatic amino acids also serve as precursors for several plant hormones including salicylic acid (SA) that plays a critical role in plant defense. A branch point intermediate of this pathway is chorismate and its partitioning towards various products is mediated by several chorismate metabolizing enzymes including chorismate mutase (CM), that converts chorismate to prephanate. $U$. maydis can "hijack" the host plant shikimic pathway to establish infection by secreting its CMs into maize cells. During the early stages of infection, CMU1 is delivered into maize cytoplasm where it binds and presumably manipulates the activities of host cytoplasmic CM. This results in increasing the flow of chorismate from the plastid to the cytosol and, in turn, lowering the available substrate for the biosynthesis of salicylic acid. Thus CMU1 effectively reduces salicylic acid synthesis and decreases the levels of this key plant defense hormone. These led to the idea that CM may serve as a general marker for biotrophy. Our results strongly support the presence of a biotrophic phase during $S$. sclerotiorum infection.

We have characterized the Sclerotinia effector, SsCM1, and have shown that inactivation of Sscm1 does not affect fungal vegetative growth, sclerotial development, or oxalic acid production, but does result in reduced fungal virulence. We have shown that, unexpectedly, SsCM1 translocates to the host chloroplast, and demonstrate that this process is required for full fungal virulence. We have also determined that the secreted SsCM1 can form heterodimers with Arabidopsis CMs. Moreover, SsCM1 is able to suppress at least some of the effects imposed by $\mathrm{H}_{2} \mathrm{O}_{2}$ in yeast and in plants.

Other achievements obtained during this joint project include demonstrating that tipping the balance between apoptosis and autophagy can dictate the outcome of SclerotiniaArabidopsis interaction. Programmed cell death (PCD) can mediated both resistance and susceptible responses during plant-microbe interactions. Apoptosis and autophagy are two principle cell death widely characterized in both animal and plant systems. S. sclerotiorum 
induces an apoptotic spreading cell death (apoptosis), via the secreted non-selective virulence determinant oxalic acid (OA); while the OA-deficient fungal mutant A2 is nonpathogenic and exhibits the restricted autophagic cell death (autophagy) in Arabidopsis. Thus, apoptosis turns out to be a killer and autophagy is a defense response. To examine whether the control of cell death can lead to opposing ends, we treated plant with chemical inducers or inhibitors before pathogen challenge and showed that induction of autophagy by Tunicamycin (TM) could inhibit the initiation of apoptosis and trigger resistance to wild-type S. sclerotiorum. Based on our data, we suggest that tipping the balance between apoptosis and autophagy can dictate the outcome of Sclerotinia-Arabidopsis interaction.

In addition to focusing on answering the questions posed in the proposal (and those arising from them), we have also been investing time in developing additional tools for studying S. sclerotiorum. CRISPR/Cas9 is a game changing gene-editing technique that can be utilized to target and modify eukaryotic genomes with exquisite accuracy. Given the fact that S. sclerotiorum grows as multinucleated cells, developing an efficient CRISPR/Cas9based genome editing technique in S. sclerotiorum will augment research in this important pathogen. By modifying a CRISPR/Cas9 system that has been successfully used for Arabidopsis genome editing, we developed a system that can correctly deliver Cas9 proteins into poly-nucleuses and to precisely express of functional sgRNA in $S$. sclerotiorum. As a proof-of-concept, the S. sclerotiorum oxalic acid (OA) key biosynthesis enzyme-encoding gene, $O A H$, has been edited using the CRISPR/Cas9 system that we developed. 


\section{Summary Sheet}

\section{Publication Summary}

\begin{tabular}{|c|c|c|c|}
\hline PubType & IS only & Joint & US only \\
\hline Reviewed & 0 & 1 & 2 \\
\hline
\end{tabular}

\section{Training Summary}

\begin{tabular}{|r|c|c|c|c|}
\hline \multicolumn{1}{|c|}{ Trainee Type } & Last Name & First Name & Institution & Country \\
\hline Postdoctoral Fellow & Li & Yurong & Texas A\&M \\
\hline Ph.D. Student & Aharoni & Liran & Hebrew University of Jerusalem & Israel \\
\hline M.Sc. Student & Tal & Liran & Hebrew University of Jerusalem & Israel \\
\hline Postdoctoral Fellow & Jiang & Maofeng & Texas A\&M & USA \\
\hline Postdoctoral Fellow & Solomon & Eitan & HUJI & Israel \\
\hline
\end{tabular}




\section{Collaboration}

We have worked together and in parallel in order to advance our goals. This has included sharing of strains and technical advice (including troubleshooting with regard to some of the strains) as well as planning future research (including the drafting of a joint manuscript). We have been in continuous contact (email/skype) in order to coordinate work and plans for the future. We have met during 2017 and then again, briefly, in 2018, shortly before Marty Dickman passed away. 


\section{Achievements}

Sclerotinia sclerotiorum is a filamentous fungus (mold) that causes plant disease. It has an extremely wide range of hosts (>400 species) and causes considerable damage (annual multimillion dollar losses) in economically important crops. It has proven difficult to control (culturally or chemically) and host resistance to this fungus has generally been inadequate. It is believed that this fungus occurs in almost every country. Virulence of this aggressive pathogen is bolstered by a wide array of plant cell wall degrading enzymes and various compounds (secondary metabolites) produced by the fungus.

It is well established that plant pathogenic fungi secrete proteins and small molecules that interact with host cells and play a critical role in disease development. Such secreted proteins have been collectively designated as "effectors". Plant resistance against some pathogens can be mediated by recognition of such effectors. Alternatively, effectors can interfere with plant defense. Some such effectors are recognized by the host plant and can culminate in a programmed cell death (PCD) resistant response.

During the course of this study, we analyzed an effector in Sclerotinia sclerotiorum. This specific effector, SsCM1 is the protein chorismate mutase, which is an enzyme involved in a pathway which is important in the production of important amino acids, such a Tryptophan. We have characterized the Sclerotinia effector, SsCM1, and have shown that inactivation of Sscm1 does not affect fungal vegetative growth, development or production of oxalic acid (one of this fungus' secondary metabolites associated with disease) production. However, yhis does result in reduced fungal virulence. We show that, unexpectedly, the SsCM1 protein translocates to the host chloroplast, and demonstrated that this process is required for full fungal virulence. We have also determined that the fungal SsCM1 protein can interact with similar proteins produced by the host. In addition, we have shown that the fungal SsCM1 is able to suppress at least some of the effects imposed by reactive oxygen species which are produced as a defense mechanism by the host. Last, but not least, the results of our studies have provided evidence contradicting the current dogma on at least some of the mechanist aspects of how this pathogen infects the host. Contrary to previous 
assumptions, indicating that this pathogen kills its host by use of metabolites and enzymes that degrade the host tissue (a process called necrotrophy), we now know that at least in the early phases of infection, the fungus interacts with live host tissue (a phenomenon known as biotrophy). Taken together, the results of our studies provide novel insights concerning the mechanistic aspects of Sclerotinia-host interactions. We hope this information will be used to interfere with the disease cycle in a manner that will protect plants from this devastating fungus. 


\section{Changes/modification in the research program.}

During the first year of the project, and to a certain extent the second, we encountered some technical difficulties, mainly with regard to the stability of some of the original mutants produced. These have not deterred us from continuing to pursue the specific goals of our research project. However, and in line with data we had accumulated, we expanded our analysis of Sclerotinia-host interactions to include more in-depth analysis of cell death (apoptotic and autophagous events) as a part of the fungus host interaction. We have also, in our tradition, invested time and efforts in expanding the array of methods and techniques (e.g., CRISPR/Cas9) for studying this important pathogen. 
Publications for Project US-4833-15C

\begin{tabular}{|c|c|c|c|c|c|c|}
\hline $\begin{array}{l}\text { Stat } \\
\text { us }\end{array}$ & Type & Authors & Title & Journal & $\begin{array}{l}\text { Vol:pg } \\
\text { Year }\end{array}$ & $\begin{array}{c}\text { Cou } \\
\text { n }\end{array}$ \\
\hline Published & Reviewed & $\begin{array}{c}\text { Li, Y. R., Kabbage, } \\
\text { M., Liu, W., and } \\
\text { Dickman, M. B. }\end{array}$ & $\begin{array}{l}\text { Aspartyl protease mediated } \\
\text { cleavage of At-BAG6 is } \\
\text { necessary for autophagy and } \\
\text { fungal resistance in plants }\end{array}$ & The Plant Cell & $\begin{array}{l}28: 233- \\
2472016\end{array}$ & US only \\
\hline Published & Reviewed & $\begin{array}{l}\text { Martin Dickman, } \\
\text { Brett Williams, } \\
\text { Yurong Li, Paul } \\
\text { Figueiredo and } \\
\text { Thomas Wolpert }\end{array}$ & Reassessing apoptosis in plants & Nature Plants & : 2017 & US only \\
\hline Published & Reviewed & $\begin{array}{l}\text { Kabbage, M., } \\
\text { Yarden, O. and } \\
\text { Dickman, M.B. }\end{array}$ & $\begin{array}{c}\text { Dining habits of Sclerotinia } \\
\text { sclerotiorum: switching from } \\
\text { biotrophic to necrotrophic } \\
\text { lifestyle }\end{array}$ & Plant Science & $\begin{array}{l}233: 53- \\
602015\end{array}$ & Joint \\
\hline
\end{tabular}

